\title{
DEVELOPMENT OF PASSIVE ALIGNMENT TECHNIQUES FOR THE ASSEMBLY OF HYBRID MICROSYSTEMS
}

\author{
Christian Brecher, Martin Weinzierl and Sven Lange \\ Fraunhofer Institute for Production Technology
}

Abstract: Conventional machining methods have been developed to meet the standards of ultra precision machining. Special milling processes utilizing monocrystalline diamond tools, the so-called fly-cutting processes, are used successfully to manufacture highly precise microstructures with an optical surface finish. In micro assembly often positioning accuracies of only a few micro meters are needed. An approach of the Fraunhofer IPT is to achieve these accuracies using passive alignment strategies. In this paper, the ultra precision machining of the v-groove structures as well as their passive alignment capacities for micro assembly tasks are presented.

Key words: ultra precision machining, passive alignment, assembly, micro components

\section{INTRODUCTION}

Microsystems are often characterised with a high functional density on an area of only a few millimetres. The combination of optical, mechanical, electronic or fluidic components creates the latest generation of microsystems, the so-called hybrid microsystems. To guarantee the function of these systems, the exact cooperation of their single components is absolutely necessary. In general, this comprises the exact alignment of the components within a range of a few micrometers and below. Therefore, single item production systems are today's standard in the production of hybrid microsystems. These extensive manufacturing and assembly processes increase the cost and complicate line production. In fact, along with the handling and joining operations, the micro assembly process can account for up to $80 \%$ of the total production costs of a micro system ${ }^{1}$. The growing demand on the integration of fluidic and optical components, for instance, does not always allow for the use of silicon wafers. Therefore, existing knowledge from the silicon-wafer technology, which has been 
established over the last years for applications in microelectronics, as well as conventional production technology, can not only be used for the manufacturing of such micro systems. The production and assembly of hybrid microsystems requires appropriate handling and alignment techniques in combination with precise process control to comply with the specified narrow tolerances. Research is carried out at the Fraunhofer Institute for Production Technology (IPT), to develop cost-efficient methods to support the production, handling and assembly of micro components and -systems. In this paper, the ultra precision manufacturing of passive alignment structures as well as their potential for the passive alignment of micro components is introduced and discussed. The achievable passive alignment accuracies are discussed with the example of passive assembled glass fibre arrays.

\section{ULTRA PRECISION TECHNIQUES FOR THE PRODUCTION OF HIGHLY ACCURATE PASSIVE ALIGNMENT STRUCTURES}

Passive alignment structures consist of elements that hold the micro components in exactly defined positions. This is accomplished by an in general statically determinate embedding of the micro components in structures with narrow form tolerances and high surface qualities. The manufacturing of these structures requires highly precise machining processes. Lithographic processes in combination with etching have been established in silicone technology. Further developments, such as the LIGA process, combine multiple processes to create microstructures which are precise enough to be used for the passive alignment of micro components. However, the amount of processes involved, increases the cost and effort for the manufacturing of single passive alignment structures. Here, conventional metal cutting processes such as milling or planning offer the possibility for a cheap and fast manufacturing of microstructures. Regarding bulk production, these microstructures may be used for replication processes such as hot embossing of plastics or glass. At the Fraunhofer IPT, the machines and the processes themselves have been designed to meet the requirements of ultra precision machining. The most frequently used ultra precision machining process is fly-cutting. In this milling process, a single tool is rotating on a high precision air bearing spindle with up to $3000 \mathrm{~min}^{-1}$ and moved across the surface of the work piece with an accuracy of $0.1 \mu \mathrm{m} / 100 \mathrm{~mm}$. Thus, linear groove-structures in the shape of the projected tool geometry are cut into the work piece. The use of monocrystalline diamond as a cutting material enables the manufacturing of tools with cutting edges down to $50 \mathrm{~nm}$. These allow for a chip removal down to a thickness of only a few micrometers which results in very high surface qualities of 10-20 nm Ra.

In order to comply with the narrow form tolerances which are required for highly accurate passive alignment structures, the relative alignment between the tool and the workpiece is decisive. Therefore alignment techniques have been developed to define the tool's position exactly in respect of its six degrees of freedom. During rigging, the tool is prealigned 
with the precision of an articulated robot which has a repeatable accuracy of $\pm 30 \mu \mathrm{m}$. This repeatability is by far not enough for the required machining precision but is sufficient to position the tool within the measuring range of $480 \mu \mathrm{m} \times 360 \mu \mathrm{m}$ of a measuring device which is integrated in the ultra precision machine tool and actively detects the translational offset between the currently mounted tool and a reference (Fig. 1). The reference is a fixed point which is defined within the measurement range, usually the crosshair of the camera system. Prior to structuring, the position vector between the reference and the work piece is determined with the use of a tactile sensor. The measuring device consists of a CCD-camera combined with a telecentric objective and has a hardware resolution of $0.465 \mu \mathrm{m}$. Software is used to measure the tool's position and to calculate the offset to the reference which is then compensated within a submicrometer range by the control system of the machine tool. The accuracy of the offset detection is influenced by the positioning accuracy of the tool within the measurement range and by the measurement uncertainty of the system. The positioning of the tool is mainly influenced by the accuracy of the passive alignment of the spindle rotor, which lies within $\pm 5 \mu \mathrm{m}$. In combination with a misalignment of the camera, that is below $0.011^{\circ}$, deviations of $0.001 \mu \mathrm{m}$ result in horizontal and vertical direction. Repeated measurements of the tool tip position have revealed a maximum deviation of the measured value of $0.248 \mu \mathrm{m}$, which is mainly attributable to the measurement uncertainty of the software. Therefore, the translational offset of the tools is kept well below $0.5 \mu \mathrm{m}$. The tool's rotational positions are determined by passive alignment. The standardized design of the tool retainers enables the integration of fitting surfaces to define the tool's roll angle $\varphi$ by passive alignment (Fig. 1). The tool itself is adjusted appropriately within the retainer. Therefore the measuring device can be swayed by $90^{\circ}$ to detect all rotational displacements. Once each tool is adjusted right in its retainer it can be exchanged multiple times with much higher precision than simply mounting it in the spindle without knowing its exact position. The condition of the tool's cutting edge is examined frequently throughout the whole cutting process by use of 

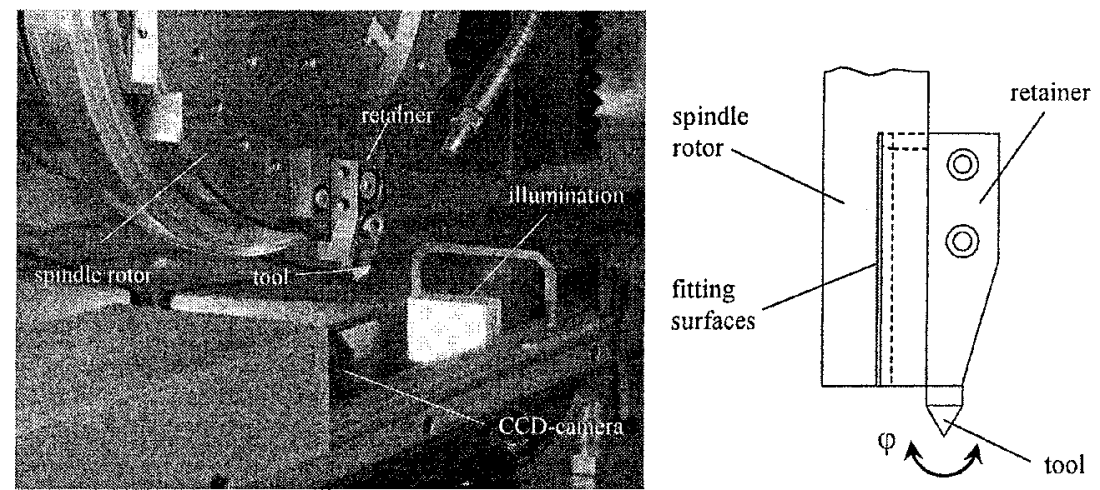

Figure 1. Optical set up (left) for the active alignment and fitting surfaces (right) for the passive alignment of the tool position

the metrology device as well. An automatic tool changer replaces the tool in case of excessive tool wear and is used to switch between different shaped tools to manufacture complex structures. The use of these innovative technologies increases the process stability of ultra precision machining and enables the manufacturing of complex structures with sub micrometer tolerances.

As it is known form conventional cutting processes, high cutting forces can cause tool deflection during the process. This effect causes form deviations and thus increases the work piece tolerances. Concerning the stiffness of the fly-cutting process, the weakest link is the air bearing of the spindle. However, with a working load below $1 \mathrm{~N}$ of the ultra precision machining process and a stiffness of the air bearing of $40 \mathrm{~N} / \mu \mathrm{m}$, the maximum tool deflection would amount for $0.025 \mu \mathrm{m}$. Therefore, the influence of cutting forces in ultra precision fly-cutting can be neglected regarding the tolerances of microstructures.

\section{PASSIVE ALIGNMENT OF GLASS FIBRES}

The focal point of the research work at Fraunhofer IPT on the passive alignment of micro components is the analysis of the entire chain of tolerances resulting from the manufacturing and assembly processes. Besides the macroscopic geometry of the alignment structures, the machine and process accuracy during the fabrication of the structures as well as the tolerances of the micro components themselves have an influence on the net accuracy of the passive alignment. For a precise and accurate analysis of the passive alignment structures and the alignment process itself, the use of highly precise micro components is necessary. Single mode glass fibres which are used for the transmission of optical signals have very little form tolerances regarding variations in diameter $( \pm 0.7 \mu \mathrm{m})$, roundness $(\leq 0.7 \%)$ and core eccentricity $(\leq 0.5 \mu \mathrm{m})^{2}$. This is the reason, why the experimental 
research described in the following was carried out on the example of the passive alignment of glass fibres.
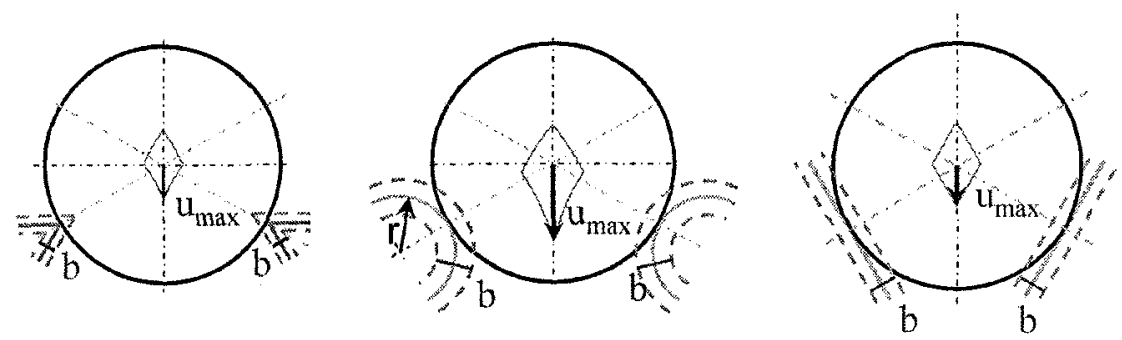

Figure 2. Models for the theoretical analysis of pointed, round and flat passive alignment features

First of all, the theoretical positioning accuracy of glass fibres in passive alignment structures was derived with the help of mathematical models. For this analysis, general tolerance ranges for the glass fibres (a) and the alignment structures (b) were taken from the DIN ISO 1101 standards $^{3}$. In the theoretical analysis, round, pointed and flat passive alignment features were compared regarding their precision in the passive alignment of cylindrical microcomponents (Fig. 2). The flat alignment features were identified to have the best alignment accuracy. Therefore v-groove structures were chosen as passive alignment structures since they can be manufactured easily through ultra precision machining by means of fly-cutting.

Considering the tolerance ranges of the glass fibres (a) and the passive alignment structure (b) a maximum horizontal deviation of the centre points of the glass fibres of

$$
u_{\max , x}=\frac{a+b}{2 \cdot \cos (\alpha / 2)}
$$

and a maximum vertical deviation of the centre points of the glass fibres of

$$
u_{\max , y}=\frac{a+b}{2 \cdot \sin (\alpha / 2)}
$$

can be derived. 
The total tolerances of the glass fibres position add up to approximate $2.0 \mu \mathrm{m}$ for the centre point positions of the glass fibres. A tolerance range of $1 \mu \mathrm{m}$ (peak-to-valley) for the diamond machined v-groove structures was derived from an optical profile measurement, using a confocal microscope, which is shown in Fig. 3. With examples (1) and (2), the resulting theoretical positioning errors for the glass fibre centre points in v-grooves with opening angles of $60^{\circ}, 90^{\circ}$ and $120^{\circ}$ can be calculated (Table 1 ).

Table I. Maximum positioning errors of the glass fiber center points during the alignment of glass fibers in v-groove shaped passive alignment structures with different opening angles

\begin{tabular}{llll}
\hline & $60^{\circ}$ & $90^{\circ}$ & $120^{\circ}$ \\
\hline $\mathrm{u}_{\max , \mathrm{x}}$ & $1.7 \mu \mathrm{m}$ & $2.1 \mu \mathrm{m}$ & $3.0 \mu \mathrm{m}$ \\
$\mathrm{u}_{\max , \mathrm{y}}$ & $3.0 \mu \mathrm{m}$ & $2,1 \mu \mathrm{m}$ & $1.7 \mu \mathrm{m}$ \\
\hline
\end{tabular}

Following the systematic geometric analysis of the resulting tolerance fields for the passive alignment of the glass fibres, the achievable positioning accuracy was experimentally investigated at Fraunhofer IPT. For these experiments, test structures were made of aluminium. First the main structure body was machined with a 5-axis micro milling operation and then $40 \mathrm{v}$-grooves with varying apex angles $\left(60^{\circ}, 90^{\circ}, 120^{\circ}\right)$ and a spacing of $250 \mu \mathrm{m}$ were introduced via ultra precision machining (fly-cutting) using monocrystalline diamond tools.

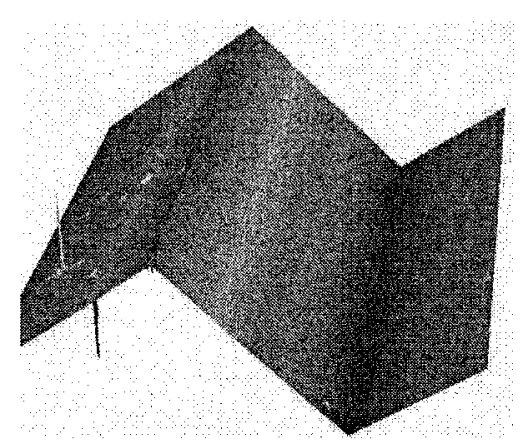

three-dimensional profile plot

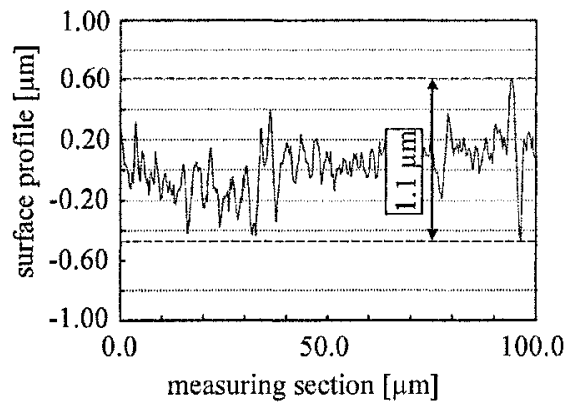

Figure 3. Three-dimensional confocal white light profile plot (left) with the derived twodimensional profile section (right)

The glass fibres were then manually prealigned within the v-grooves with the help of a stereo microscope and slightly pressed into the alignment structures by means of a high-precision, micro milled clamping device. During this mounting process, the glass fibres were set against a diamond-turned limit block, in order to guarantee that the fibre end surfaces 
flushed with the end surface of the alignment structure. In order to compensate for the variability in the fibre diameters and to press each of the 40 fibres into the v-grooves with the same force, a clamping block with a $70 \mu \mathrm{m}$ thick layer of latex was utilised. Figure 4 shows a scanning electron microscope (SEM) image of a section from the one-dimensional glass fibre array.

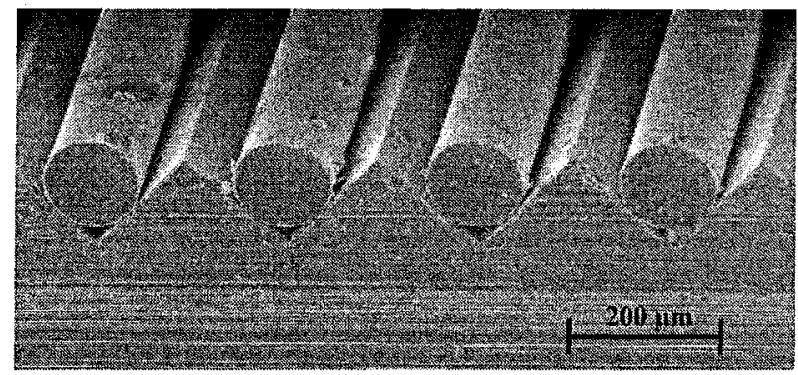

Figure 4. SEM image of passive aligned glass fibers (100x)

The measurement of the relative positioning accuracy of the core centre points of the glass fibres was carried out with a high-resolution image processing system ${ }^{4-6}$. To ensure the highest possible contrast to their surrounding, cold cathode light was fed into the fibres' free ends. In this way the fibre cores were illuminated by the light conducted through the glass fibres only. For the interpretation of the image processed measurement data, the corresponding Gaussian distributions and standard deviations $\left(\sigma_{x}, \sigma_{y}\right)$ of the $x-y$-pairs of the fibre core centre points were determined. By means of the standard deviations the three-dimensional Gaussian distribution of the fibre centre points can be expressed as follows:

$$
P(x, y)=\frac{1}{2 \cdot \pi \cdot \sigma_{x} \sigma_{y}} \cdot \exp \left(-\frac{x^{2}}{2 \cdot \sigma_{x}^{2}}-\frac{y^{2}}{2 \cdot \sigma_{y}^{2}}\right)
$$

Figure 5 shows the three-dimensional Gaussian distribution of the fibre centre points positions in the section plane of the passive alignment structures. The z-axis of the Graphs represents the frequency density of the Gaussian distribution. The outline of the top-views represents the 3- $\sigma$-line. According to the $3-\sigma$-criteria, $99.7 \%$ of the fibres centre points were positioned with a maximum error of $+1-3.2 \mu \mathrm{m}$ for apex angles of the grooves of $60^{\circ},+/-3.6 \mu \mathrm{m}$ for apex angles of $90^{\circ}$ and $+/-3.9 \mu \mathrm{m}$ for apex angles of $120^{\circ}$. 

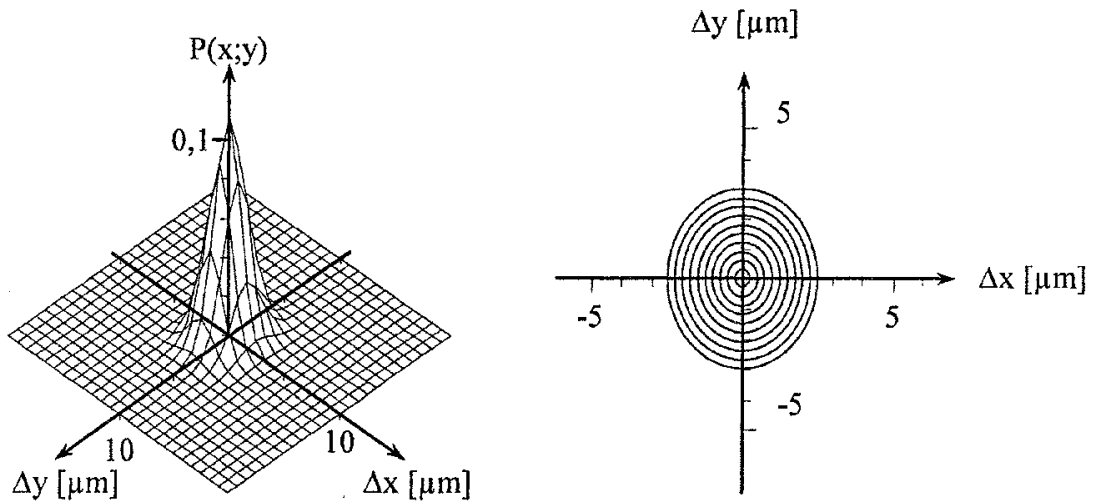

Figure 5. Tree-dimensional Gaussian distribution to evaluate the positioning accuracy of the passive alignment of glass fibers in v-grooves

The measured values lie substantially above the theoretical values which can be traced to the following influential factors. In the experimental research a maximum difference of $2.2 \mu \mathrm{m}$ was detected to the theoretically determined deviations of the fibre core centre points (see Table 1). The accuracy of the image processing is one of the factors which influence the experimental results. The image processing system provides a resolution of a $0.5 \mu \mathrm{m}$ per pixel upon the entire field of view. A software interpolation between individual pixels enhances the resolution by a factor of ten during the measurement process. Thereby measurements with a theoretical resolution of up to $0.1 \mu \mathrm{m}$ can be done. Measurement errors occur here, for example from stray light which hindered the precision of the imaging of the fibre core contour. Additionally, errors are caused by the interpolation of the circular contour of the fibre cores and the centres of the circles. The choice of the threshold values for the binary filter also plays an important role. A further but rather minor $(<100 \mathrm{~nm})$ influence on the experimental results is the positioning accuracy of the $z$-axis of the positioning system which is used to position the glass fibres within the measurement range of the camera system.

Assuming, that $0.2 \mu \mathrm{m}$ of the measured difference form the theoretical values can be assigned to the influences described above, the greatest influence on the experimentally determined positioning accuracy is due to the contamination of the glass fibres and alignment structures with coating remains and atmospheric dust. According to the deviations of the fibres core centre points which were determined in the experimental analysis, these account for a total of $2 \mu \mathrm{m}$

Recapitulatory it is safe to say, that passive alignment structures which are manufactured by ultra precision machining processes like fly-cutting are well suited for a high-precision passive alignment of glass fibres. With an optimization of the cleaning methods for the stripped glass fibre ends and for 
the ultra precise microstructures which are used for the passive alignment as well a minimization of the failure influences on the used measuring system, positioning tolerances of down to $+/-1 \mu \mathrm{m}$ should be attainable within the passive alignment of glass fibres in ultra precision machined v-groove structures.

\section{CONCLUSION}

For the passive alignment of glass fibres in v-groove structures, the alignment of 40 fibres in a one-dimensional array resulted in a best positioning accuracy between the centre points of the fibre cores of $+/$ $3.2 \mu \mathrm{m}$ in v-grooves with an apex angle of $60^{\circ}$. Slight differences were identified between the presented theoretical model and the experimental investigations. These can be attributed to contaminations of the glass fibres and the alignment structures as well as uncertainties in the measuring system. The experimental results show, that the passive alignment of glass fibres in alignment structures which are manufactured by ultra precision processes like fly-cutting using monocrystalline diamond tools can be realized with positioning accuracies of few micro meters. This shows that ultra precise manufactured passive alignment structures are well suited for lots of alignment tasks within micro assembly.

\section{OUTLOOK}

In the field of passive alignment of micro components, future research work at the Fraunhofer IPT will extend into the experimental investigation of passive alignment of spherical and cubical micro components to validate the theoretical results which have come out so far. Beyond that, theoretical as well as experimental investigations will be made on the simultaneous passive alignment of multiple micro parts and the handling of such arrangements of micro parts. The results form these researches are expected to be suitable for the fully automated mounting of hybrid microsystems.

\section{ACKOWLEDGEMENTS}

The achievements presented in this papers are the results from the publicly funded research projects GroßMikro (AiF / BMWA), SFB 440 (DFG) as well as the EC-funded Network of Excellence 4M. The authors would like to thank the AIF, the DFG and the European Commission for their support which enabled the work done in the field of ultraprecision and microsystem technology.

\section{REFERENCES}

1. S. Koelemejer Collet, J. Jacot: Cost Efficient Assembly of Microsystems. In: MST News, No. 1 (9), 1999, p. 30-32

2. Corning Incorporated: Corning SMF28 Optical Fiber, Product Information, 2004 
3. N.N.: Norm DIN ISO 1101: Form- und Lagetolerierung. Form-, Richtungs-, Orts- und Lauftoleranzen, Deutsches Institut für Normung e.V., 1985

4. C. Demant, B. Streicher, A. Waszkewitz: Industrielle Bildverarbeitung, Springer, 1998

5. B. Jähne: Digitale Bildverarbeitung, Springer, 1989

6. K. Hentschel, M. Wendelstein: Telezentrische Objektive fur die industrielle Bildverarbeitung, Stemmer, 2002 